reduction and stable retention, and in the series of twenty-three cases recorded here he has shown how this particular obstacle can be eliminated through a comparatively limited arthrotomy approach. His results are of course not yet long-term. The masterly analysis by Muller and Seddon of the late results of the treatment of congenital hip dislocations at the Royal National Orthopaedic Hospital over a period of fifty years is not only of intrinsic historical interest, but provides us with information of great significance. Their verdict that "there was reason for sober satisfaction with the results of closed reduction" is of prime importance in determining our future method of attack on dislocations in young children. It would appear that our aim should be to define as far as possible the special morphological features of each dislocation before attempting any type of reduction. This should enable us to recognise those cases in which closed reduction, whether by frame traction or gentle manipulation, is likely to fail completely or to result in an incongruent joint. Such cases will no doubt form a minority of the dislocations in young children; but in this group the technique of open reduction must be available.

Until we are able to diagnose and treat the dislocations in the first year of life, it is wise that we should not be tempted to adopt a perfectionist attitude in dealing with what is still a difficult problem. For as Muller and Seddon have pointed out, the "life" of a successfully reduced hip is about twenty-five years. On the other hand, the Royal National Orthopaedic Hospital follow-up has also reminded us that many adult patients with unreduced dislocations are able to lead an active and relatively comfortable existence. Such patients therefore should not become the victims of light-hearted surgical adventures.

HaRry Platt.

\title{
THE VASCULARISATION OF BONE
}

Investigations of the vascularisation of organs and tissues are useful from at least two standpoints. The normal distribution of vessels, apart from its purely anatomical interest, may be correlated with function (as shown, for example, by the work on the renal circulation carried out at the Nuffield Institute for Medical Research), or may assist the surgeon in evaluation of techniques. The examination of arterial anastomoses around or within the tissue is of importance for a comprehension of the efficiency of the collateral circulation which may be established during surgical procedures. In general, organs of endodermal origin, such as the stomach, intestine, thyroid, urinary bladder and pancreas, appear to be provided with particularly rich arterial anastomoses, but organs and tissues developed from mesoderm vary considerably in their reaction to ischaemia from the interruption of individual arteries of supply. The nearer to an organ an artery of supply is interrupted, the fewer the arterial anastomoses and the greater the likelihood of damage to the organ. Interruption of periosteal vessels, for example, would be more likely to lead to bone necrosis than occlusion of the common iliac artery, and it can be shown that interruption of the common iliac artery in the rat leads to no obvious change in the radiological appearance of the head of the femur up to four months after operation. The vascularisation of bone after periosteal stripping, or obstruction of individual periosteal vessels, would depend largely on the efficiency of intra-osseous anastomoses; but this particular aspect of the vascularisation of bone has led to many divergent statements in the literature. The investigation of the vascularisation of the head of the femur by Trueta and Harrison in this number of the Journal is therefore most welcome.

The arteries supplying the head of the femur are well known to the anatomist. The medial circumflex femoral artery in its course behind the neck of the femur gives off several periosteal branches, anastomoses with vessels from the posterior branch of the obturator artery and may furnish an artery passing along the ligamentum teres, or even entirely supplant the acetabular twig from the posterior branch of the obturator artery which is usually 
considered to course along this ligament. Finally, the ascending branch of the medial circumflex femoral artery undergoes anastomosis with branches of the superior and inferior gluteal arteries in the region of the trochanteric fossa. On these grounds alone, therefore, the medial circumflex femoral artery should be more respected than the acetabular branch of the obturator. In addition, however, it is now clear that the medial femoral circumflex artery supplies most of the epiphysis and the whole of the metaphysis by way of its metaphysial and lateral epiphysial branches. A rich arterial anastomosis between the lateral and medial epiphysial arteries has also been clearly demonstrated (Figs. 3 and 23 ); the degree of efficiency of this anastomosis must obviously be considered in all cases of adduction fracture of the neck of the femur, but such an anastomosis is also of importance in the collateral circulation which may be formed as a result of arterial occlusion. Thus Olovson (Acta Chirurgica Scandinavica, 1941, 86, Supp. 67) has shown that intra-osseous vessels as well as the periosteal network of vessels and the capsular arteries may show considerable dilation after ligation of the external iliac artery in the rabbit. The collateral channels that develop after such operations may be sinuous.

We are reminded of the mechanism of articular cartilage nutrition, since Trueta and Harrison confirm the existence of a subchondral circulation. Present anatomical opinion is in favour of nutrition from synovial fluid and, at the periphery of joint cartilage, by the circulus articuli vasculosus. Ekholm (Acta Anatomica, 1951, 11, Supp. 15-2), however, from his studies with ${ }^{198} \mathrm{Au}$, found that nutrition of rabbit joint cartilage most probably takes place partly from synovial fluid and partly via the direct contacts between subchondral vascular channels and the basal layers of the cartilage, but he was unable to establish the quantitative relation between these two methods. It would be unusual if such well demarcated subchondral vessels did not have some nutritional function, however small. The relationships on the two surfaces of the articular cartilage are almost akin to those seen in the varying morphological types of chorio-allantoic placentation, and Flexner and Gellhorn (American Journal of Obstetrics and Gynecology, 1942, 43, 965) have shown that the smaller the number of tissue layers (including the endothelium of blood vessels) between maternal and foetal circulations, the greater the rate of transfer of radioactive sodium across a unit weight of placenta.

An anastomosis of intra-osseous vessels supplying the metaphysis and epiphysis of the head of the femur was described by Sappey 1873 (quoted by Poirier 1896), but refuted by Poirier (Traité d'Anatomie Humaine, 1896, tome II, fasc. II, p. 825) and others. There have been similar divergent opinions regarding the presence or absence of the arteria ligamenti teretis, and the extent of the vascularisation of the femoral head by this artery when present. It was once argued by Walmsley (Journal of Anatomy and Physiology, 1915, 49, 434) that, since the femoral head undergoes "no especial atrophy" in the absence of the ligamentum teres in congenital dislocation of the hip, it cannot be held that the round ligament conveys a blood supply to the head of the femur. In cases of traumatic dislocation, however, atrophy does occur in at least a proportion of cases. These new findings open the way to a better understanding of such changes, and it may well be found that a further study of the vascularisation of the upper end of the femur in congenital types of dislocation may yield information of value concerning its etiology.

It is fortunate that we now possess such excellent methods as arteriography, which can be used experimentally to enable a precise examination of vascular patterns with facility, and the production of clear and decisive illustrations such as those shown by Trueta and Harrison. Arteriography has also been used to great advantage in the examination of the blood supply of the femoral shaft by $\mathrm{Mr}$ Gowans Laing as reported in his paper in this number of the Journal. The variations that exist in the nutrient arteries of bone are insufficiently recognised and must influence orthopaedic practice, and it is to be hoped that an extensive examination of other regions may be initiated.

R. G. HARRISON.

vol. 35 B, No. 3, AUgust 1953 